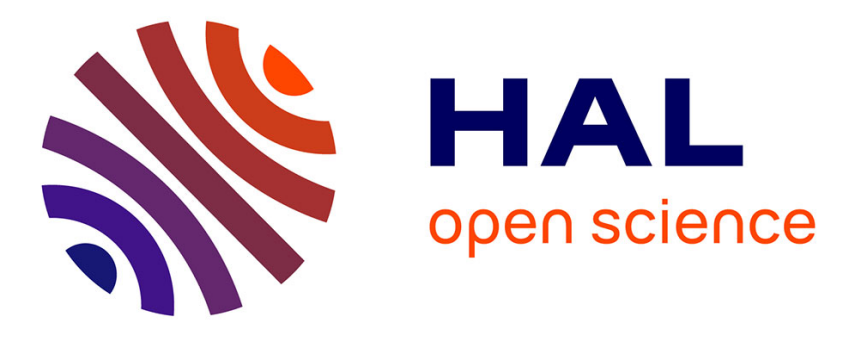

\title{
Long-time properties of trapping on fractals
}

\author{
J. Klafter, G. Zumofen, A. Blumen
}

\section{To cite this version:}

J. Klafter, G. Zumofen, A. Blumen. Long-time properties of trapping on fractals. Journal de Physique Lettres, 1984, 45 (2), pp.49-56. 10.1051/jphyslet:0198400450204900 . jpa-00232307

\section{HAL Id: jpa-00232307 https://hal.science/jpa-00232307}

Submitted on 1 Jan 1984

HAL is a multi-disciplinary open access archive for the deposit and dissemination of scientific research documents, whether they are published or not. The documents may come from teaching and research institutions in France or abroad, or from public or private research centers.
L'archive ouverte pluridisciplinaire HAL, est destinée au dépôt et à la diffusion de documents scientifiques de niveau recherche, publiés ou non, émanant des établissements d'enseignement et de recherche français ou étrangers, des laboratoires publics ou privés. 


\title{
LE JOURNAL DE PHYSIQUE-LETTRES
}

J. Physique - LETTRES 45 (1984) L-49 - L-56

15 JANVIER 1984, PAGE L-49

Classification

Physics Abstracts

$05.40-72.80 \mathrm{~N}-66.30$

\section{Long-time properties of trapping on fractals}

\section{J. Klafter}

Corporate Research Science Laboratories, Exxon Research and Engineering Company, P.O. Box 45, Linden, N.J. 07036, U.S.A.

\section{G. Zumofen}

Laboratorium für Physikalische Chemie, ETH-Zentrum, CH-8092 Zürich, Switzerland

and A. Blumen

Lehrstuhl für Theoretische Chemie, Technische Universität München, D-8046 Garching, F.R.G.

(Reçu le 10 octobre 1983, accepté le 24 novembre 1983)

\begin{abstract}
Résumé. - Nous étudions la probabilité de survie aux temps longs d'un marcheur aléatoire qui est capturé par le premier piège qu'il rencontre. Nous considérons aussi bien des structures régulières que fractales et nous trouvons dans le cas d'une exploration compacte que la loi asymptotique de survie est donnée par $\Phi \sim \exp \left[-C t^{\alpha}\right]$ avec $\alpha \equiv \tilde{d} /(\tilde{d}+2)$ où $\tilde{d}$ indique la dimension spectrale. Les simulations numériques donnent des résultats compatibles avec cette forme de $\Phi$, mais indiquent aussi des valeurs de $\alpha$ plus grandes que prédites dans les cas d'un réseau carré et du tamis de Sierpinski avec $\tilde{d}=1,365$.
\end{abstract}

\begin{abstract}
We investigate the long-time decay behaviour of a nearest-neighbour random walker which gets trapped at the first encounter of a sink. We consider both regular and fractal lattices and establish for compact exploration the asymptotic decay $\Phi \sim \exp \left[-C t^{\alpha}\right]$ with $\alpha \equiv \tilde{d} /(\tilde{d}+2)$ where $\tilde{d}$ is the spectral dimension. The numerical simulations support the $\Phi$ structure, but with a larger $\alpha$ for both the square lattice and the $\tilde{d}=1.365$ Sierpinski gasket.
\end{abstract}

\section{Introduction.}

The problem of the trapping of an elementary excitation by randomly distributed static traps, and especially the long time behaviour of the decay, have recently attracted considerable attention [1-4]. The fundamental observation is, as noticed earlier by Balagurov and Vaks [5], that the time-dependence of the trapping process does not follow an exponential decay law; the long-time 
decay should rather obey the relation :

$$
\Phi(t) \approx \exp \left(-C t^{\alpha}\right)
$$

where the constant $C$ includes the dependence on the trap-concentration and $\alpha$ is dimensiondependent ; from references [3] and [4] $\alpha$ is given by $d /(d+2)$.

For the one-dimensional case $[1,5]$ the region in which $\alpha$ is approximately $1 / 3$ is reached rather quickly (vide infra, Sect. 3); in fact such a regime has been recently observed by Seiferheld $e t$ al. in their measurements of electric-field dependent charge-carrier trapping [6]. On the other hand, our analysis for trapping on three-dimensional lattices show that deviations from exponentiality are small in the decay range accessible to experiments $[2,7]$; we encounter thus the situation that although the decay is asymptotically bounded by (1) $[3,4]$, the result is somewhat academic for $d=3$, since it may well remain unobservable. In two-dimensions the exponential regime is small [2, 5] so that deviations from exponentiality are well-known.

In this letter we focus on the evaluation of the long-time trapping behaviour on systems of dimensionalities between one and two. For the two-dimensional case Grassberger and Procaccia [3] (besides the derivation of(1)) have shown numerically for one trap concentration that $\alpha$ lies between $1 / 2$ and 1 . In section 3 we present our results over a wide range of concentrations and for much longer walks, and show that in all cases (1) is well-satisfied, but with $\alpha$ being around 0.8 . Thus we felt it mandatory to consider systems of dimension lower than $d=2$.

Systems of non-integral dimension are the fractals which are dilatationally self-similar objects [8]. A simple class of fractal structures are the Sierpinski gaskets : their basic unit is the $d$ dimensional simplex, from which the gasket is created by repeated dilatations [8]. As discussed in this journal by Alexander and Orbach [9] and by Rammal and Toulouse [10], for an embedding Euclidean space of dimension $d$, one has to distinguish between the Hausdorff fractal dimension $\bar{d}=\ln (d+1) / \ln 2$ (which gives the site density) and the spectral dimension of the gasket $\tilde{d}=2 \times$ $\ln (d+1) / \ln (d+3)$; it is $\ddot{d}$ which determines basic dynamical aspects of the fractal [9-11]. From the last relation it is obvious that $\tilde{d}$ lies between 1 and 2 for all $d$-values, so that Sierpinski gaskets allow one to bridge the region between $d=1$ and $d=2$. We also note that fractal structures may be used to model disordered systems $[9,11-13]$ and that the results were used to explain experimental findings on naphthalene [14].

In a recent study we have addressed the problem of the decay due to capture by randomly distributed traps on the $d=2$ and $d=3$ Sierpinski gaskets [15]; there we have analysed, using a cumulant expansion on the number of distinct sites visited [16], the initial stages of the decay. In this work we extend these calculations to much longer times. The gaskets are ideally suited for such studies, since, as observed by Rammal et al. [11, 17], and by us [16], the distributions of distinct sites visited reach their asymptotic regimes quite rapidly.

In the following section we extend the analysis of references [3] and [4] to fractal structures and determine that $\alpha$ in (1) should be $\alpha=\widetilde{d} /(\tilde{d}+2)$. The derivation is facilitated by using here the concept of compact exploration, as stressed by de Gennes [13]. In section 3 our numerical results are presented both for regular $(d=1$ and $d=2)$ as well as for fractal lattices, which results are then discussed in section 4.

\section{Theoretical aspects of the decay.}

In this section we focus on the decay functions due to trapping, and take the traps to be randomly distributed on the gasket, occupying its sites with probability $p$ [16]. The microscopic transfer rates from a site to its neighbouring sites are assumed to be equal, and the walker gets trapped at the first trap encounter.

For a particular realization of the random walk on the trap-free gasket, let $R_{n}$ denote the number of distinct sites visited in $n$ steps. Note, as is usual in disordered systems, the difference from the 
regular lattice : here the stochastic variable $R_{n}$ depends both on the starting point on the gasket, and on the sequence of directions of the steps; for a regular lattice the starting point is irrelevant. For the same realization of the walk let $F_{n}$ denote the probability that trapping has not occurred up to the $n$th step in the ensemble of lattices doped with traps. Thus $F_{n}$ is also a stochastic variable, so that :

$$
F_{n}=(1-p)^{R_{n}-1}
$$

assuming the origin of the walk not to be a trap, and, in standard fashion, having $R_{0}=1$. The measurable survival probability is $\Phi_{n}$, the average of $F_{n}$ over all realizations of the random walk $[16,18]$

$$
\Phi_{n}=\left\langle F_{n}\right\rangle=\left\langle(1-p)^{R_{n}-1}\right\rangle .
$$

As mentioned, the average in (3) also includes the average over starting points, and may be viewed as a double-average; we encountered a similar situation for the continuous time random walk model [19].

In reference [15] we have used (3) to determine the decay law $\Phi_{n}$ from the distribution $R_{n}$ of distinct visited sites; the form of (3) allows also to expand $\Phi_{n}$ in terms of the cumulants of $R_{n}$. As shown, the first two cumulants describe very well the decay for short and medium long times [15]. Here we are interested in the long-time regime, so that we will treat the decay in a different manner.

Let us start by noticing that on a gasket the exploration is compact, in the sense of de Gennes [13, 20]. Already visited sites have a high probability of revisitation, so that, given a compact volume $V$ which contains the walker, most points inside $V$ are visited before a new site outside the volume is explored. An exact example for compact visitation is, of course, the one-dimensional walk with nearest-neighbour steps. Thus, if there is a trapping site inside $V$ and the exploration is compact the survival probability is negligible.

For a given trap distribution around the origin of the walk there is a maximal trap-free volume $V$. Following Lifshitz [21] and Balagurov and Vaks [5] (similar ideas are echoed in references [3, 4, 22]) we observe that the decay function is determined by the solution of the diffusion equation in $V$ with absorbing boundaries, so that the long-time behaviour is given by the lowest eigenvalue $\varepsilon(V)$, and goes as $\mathrm{e}^{-t \varepsilon(V)}$. The probability that the volume is trap-free is

$$
(1-p)^{V} \approx \mathrm{e}^{-p V}
$$

where we take the trap concentration to be small. To (5) corresponds the normalized Hertz-distribution $p \exp (-p V)$. Averaging the decay over all compact volumes $V$ one has asymptotically, for $t$ large :

$$
\Phi(t) \sim\left\langle\mathrm{e}^{-t \varepsilon(V)}\right\rangle_{V} \approx \int p \mathrm{e}^{-p V} \mathrm{e}^{-t \varepsilon(V)} \mathrm{d} V .
$$

We remark that (5) could also be inferred from (3) by relating $R_{n}$ to the compact, in $n$-steps visited volume $V_{n}$; one has then to consider the fluctuations of this volume as a function of $n$ : $\Phi_{n} \sim\left\langle\exp \left(-p V_{n}\right)\right\rangle$. Through (5) the dynamical decay law is expressed in terms of the geometrical volumes $V$; this allows now for fractals a straightforward scaling analysis. The volume of a fractal changes under dilatation by $\lambda$ as [8] :

$$
V(\lambda) \sim \lambda^{\bar{d}} V(1)=C_{1} \lambda^{\bar{d}} .
$$

On the other hand, phonon modes scale as [9-12]

$$
\omega(V) \sim \lambda^{-\bar{d} / \overline{\widetilde{d}}} \omega(1)
$$


and for the eigenvalues of the diffusion (or Schrödinger) equation one has the scaling :

$$
\varepsilon(V) \sim \lambda^{-2 \bar{d} / \tilde{a}} \varepsilon(1)=C_{2} \lambda^{-2 \bar{d} \widetilde{d}} .
$$

The exponent of $\lambda$ is by a factor of 2 larger than in (7), due to the first (instead of second) time differential operator of the underlying differential equation. Inserting (6) and (8) into (5) one obtains :

$$
\Phi(t) \sim \int \mathrm{e}^{-f(\lambda)} \mathrm{d} \lambda
$$

with, up to logarithmic corrections :

$$
f(\lambda)=p C_{1} \lambda^{\bar{d}}+t C_{2} \lambda^{-2 \bar{d} / \tilde{d}} .
$$

The leading term of (9) follows through a saddle-point analysis and is proportional to $\exp \left[-f\left(\lambda_{\min }\right)\right]$, where $\lambda_{\min }$ is the minimum of $f(\lambda)$; corrections may be obtained by extensions of Watson's lemma [23]. From (10):

so that

$$
\lambda_{\min }=\left(2 t C_{2} / p \tilde{d} C_{1}\right)^{\alpha / \bar{d}} \text { with } \alpha \equiv \tilde{d} /(\tilde{d}+2)
$$

$$
\Phi(t) \sim \exp \left[-f\left(\lambda_{\min }\right)\right] \approx \exp \left[-C_{3} p^{2 /(\widetilde{d}+2)} t^{\tilde{d} /(\tilde{d}+2)}\right] .
$$

We note the disappearance of $\bar{d}$ from the final result (12), which depends only on the spectral dimension $\widetilde{\partial}$. This is intuitively obvious, since nearest-neighbour random walks depend only on the topology (i.e. connectedness) of the underlying system, and not on the density of sites (whose measure is $d$ ). The result concurs with the other findings of references $[9,10,11,15]$ which establish that for dynamical processes $\bar{d}$ is the fundamental dimension. For regular lattices the different dimensions coincide, $\bar{d}=\bar{d}=d$, and (12) reverts to the formerly presented, e.g. (1), asymptotic decays $[1,3-5,21,22]$.

\section{Numerical evaluations.}

In this section we present our numerical results for the decay laws on the linear chain, the square lattice and the Sierpinski gasket of Euclidean dimension $2(\tilde{d}=1.365)$, and check their long-time decay with respect to (1) and (12).

The one-dimensional case is in as far special as for it the decay law is known in closed form [1]; in a previous work [2] we have used the exact expression as a test for our simulation procedures and have, for $p=0.01$, indicated the range of validity of an asymptotic expression $[1,5]$ closely related to (1). To exemplify the domain of validity of (1) we start from the analytical, closed form given by Movaghar et al., (6) of reference [1], which expresses $\Phi(u)$, the Laplace-transform of $\Phi(t)$ as an infinite series of simple algebraic functions of $u$. The numerically inverted Laplace-transform gives the decay laws presented in figure 1 . Here we plot the decay up to times corresponding to $n=10^{6}$ steps, for trap concentrations ranging from $p=0.3 \%$ to $p=50 \%$. The decay is plotted logarithmically as a function of $n^{1 / 3}$. As is obvious from the figure, the linearity of $\ln \Phi v s . n^{1 / 3}$ becomes good in the decay range $\Phi \leqslant 10^{-2}$. (As shown in reference [2], in the range $10^{-2} \leqslant \Phi \leqslant 1$ the cumulant expansion is appropriate). The linearity tends to get better if $p$ increases, an interesting result which indicates that the validity of equation (1) is not limited to low $p$-values, this limitation being rather an artefact of the analytical derivation, equation (4) ff.

In our former works on three-dimensional regular lattices the decay $\Phi$ did not show any drastic deviations from exponentiality $[2,7]$. However, for an increase in dimensionality one intuitively expects the regime of validity of the asymptotic (1) and (12) to be shifted to longer times. In order 


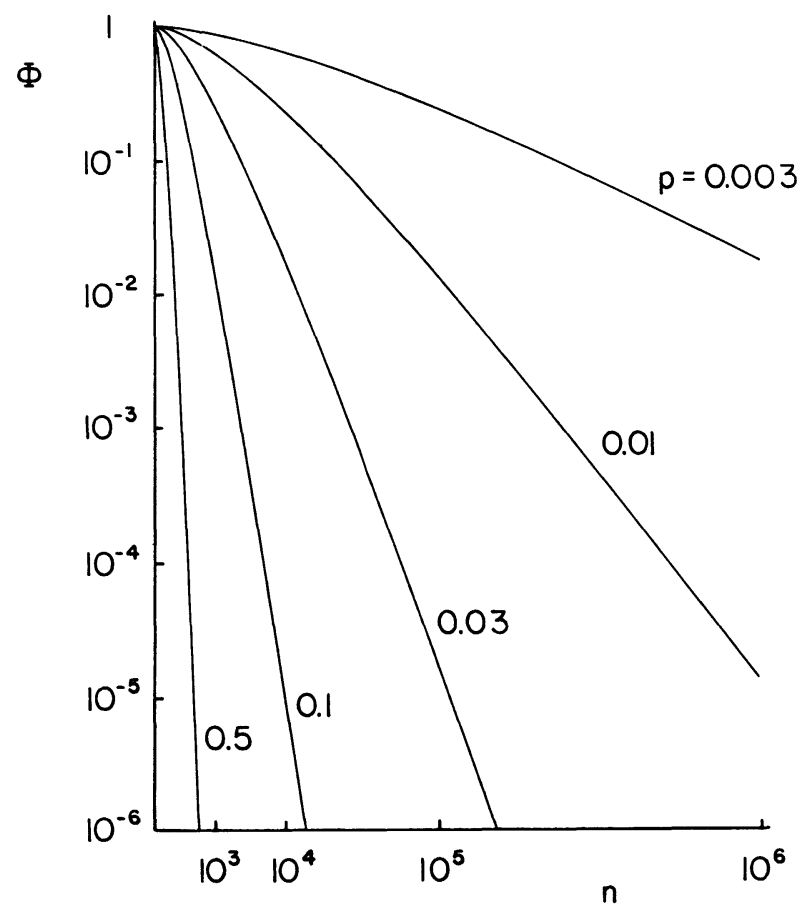

Fig. 1. - The decay law $\Phi$ due to trapping for nearest-neighbour walks on a linear chain, where $n$ is the number of steps. Plotted is $\ln \Phi v s . n^{1 / 3}$. The decay is calculated from an exact expression, reference [1], and the trap concentration varies from $p=0.003$ to $p=0.5$.

to find significant deviations we have started from the decay law on a simple square lattice. This case has also been treated in reference [3] for the trap concentration $p=1 / 8$. Here we followed the approach of our previous works $[2,7,15]$ and we evaluated the decay according to (3). We note that (3) allows, due to the preaveraging over trap positions, an increase in accuracy and a considerable decrease of numerical effort. For the determination of $R_{n} 10^{4}$ realizations of walks were used. Note that for a mean number $S_{n}$ of sites visited this corresponds to some $10^{4} .2^{S_{n}}$ walks on lattices with traps, i.e. to a very large number.

Our findings are displayed in figure 2 , where we plotted $-\ln (-\ln \Phi) v s . \ln n:$ this choice of scales allows to see directly the decay behaviour(1), which appears as a straight line. Furthermore, from the slope of the straight line the exponent $\alpha$ can be easily read-off. In figure 2 the decay laws are given for several concentrations, for $p$ ranging from $0.5 \%$ to $30 \%$. Most decays are followed over twenty orders of magnitude with the number of steps extending to $n=10^{4}$. To show the very good agreement with the calculation of Grassberger and Procaccia, we have also plotted in figure 2 their result which we have read-off from [3].

As may be seen from figure 2, the decay laws follow an almost linear pattern which proves that they may well be approximated by forms like (1). However, this behaviour is not quantitative, since some curvature is clearly observable. The slope of the curves lies higher than $1 / 2:$ we have fitted the curves of (2) both individually and collectively by straight lines over the displayed region $(10<n<10000)$; the collective fit gives the exponent $\alpha=0.78$, whereas the individual fits range from $\alpha=0.86$ for $p=0.005$ to $\alpha=0.69$ for $p=0.3$. This finding is supported by the fact that larger $p$-values render the accessible space more compact and facilitate the approach of the asymptotic expression, (1); this behaviour is also corroborated by an analysis of the $p$-dependence of $\Phi$, (12) [24]. However, from figure 2 we have to admit - even taking into account the noise of 


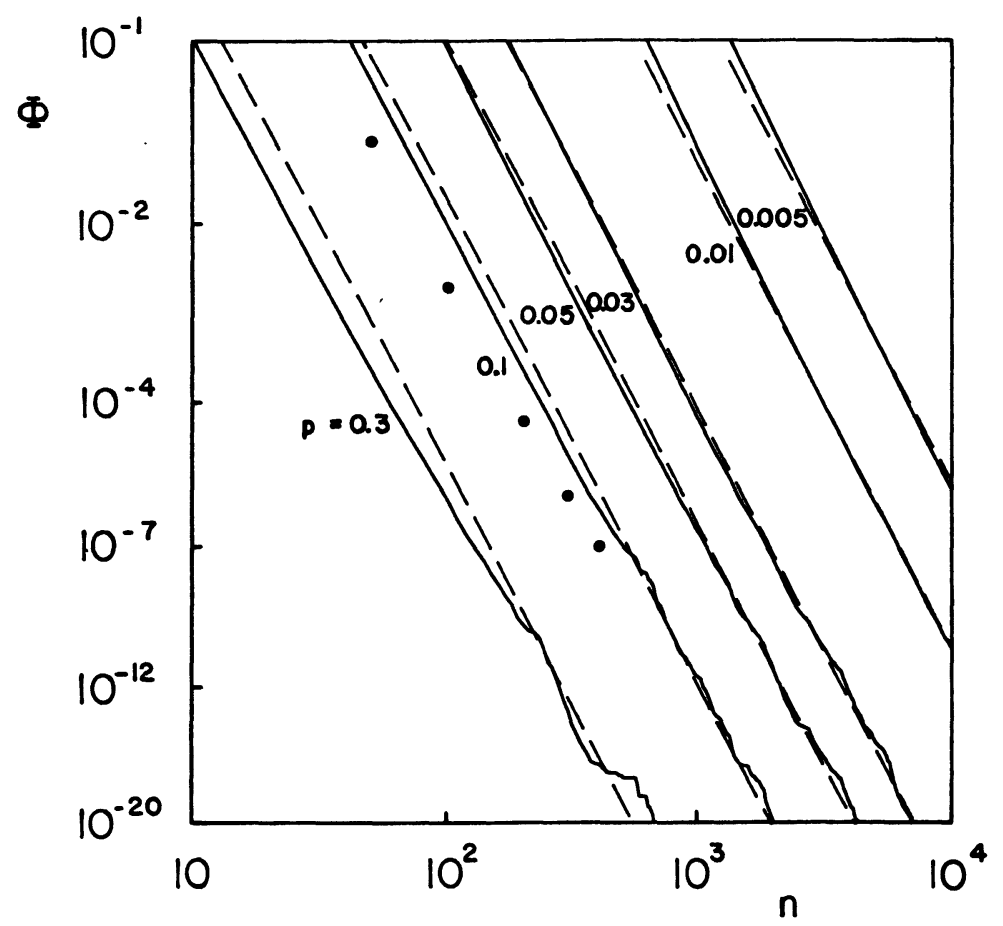

Fig. 2. - The decay law $\Phi$ for nearest-neighbour walks on a square lattice. Plotted is $-\ln (-\ln \Phi) v s$. $\ln n$, and the trap concentration varies from $p=0.005$ to $p=0.3$. The full lines are the numerically determined decay and the dashed lines are parallel straight lines giving the individual best fit. The dots denote the result of [3] for $p=1 / 8$.

the data and the fact that the curvature is determined by rare events - that we have not yet reached the asymptotic regime; also, since the curvature is small, we can't offer any guess when the exponent $\alpha=0.5$ might be attained, but we suspect it to be well outside the experimental evidence.

It is thus obvious that dimensions between $d=1$ and $d=2$ have to be explored, in order to find an $\alpha$ which complies to the Lifshitz-ideas, cf. section 2 (i.e. $\alpha=\not{d} /(\tilde{d}+2)$ ) and still lies in a range amenable to experimental observation. Fractals are thus mandatory, and we chose the Sierpinski gasket with $d=2$, i.e. $\tilde{d}=1.365$, in order to have a very small $\tilde{d}$. We have proceeded as in reference [15] and the decay laws are given in figure 3. As in figure 2 we have plotted $-\ln (-\ln \Phi) v s . \ln n$ and varied $p$ from $1 \%$ to $50 \%$.

From figure 3 one sees that the decay pattern is less linear than in figure 2 . Since curvatures are observed for all values of $p$, a fit by straight lines gives only a qualitative picture of the decay. Bearing in mind this limitation (which prevents us from extracting an exact value of the exponent $\alpha$ ), we still find that the slopes achieved are steeper than $\tilde{d} /(\tilde{d}+2)=0.406$. The overall fit leads to $\alpha=0.54$, with $\alpha$ being around 0.61 for $p=0.01$ and around 0.51 for $p=0.5$. Even concentrating on the longer times, we can read-off only values of $\alpha$ as low as 0.49 , i.e. still higher than the predicted one. Here we indeed think that lower values of $\alpha$ can be achieved by an improved numerical effort below $\Phi=10^{-20}$, but we have to admit that the quantitative observation of (12), the extension of (11) to fractal lattices, may be well outside the experimental means. 


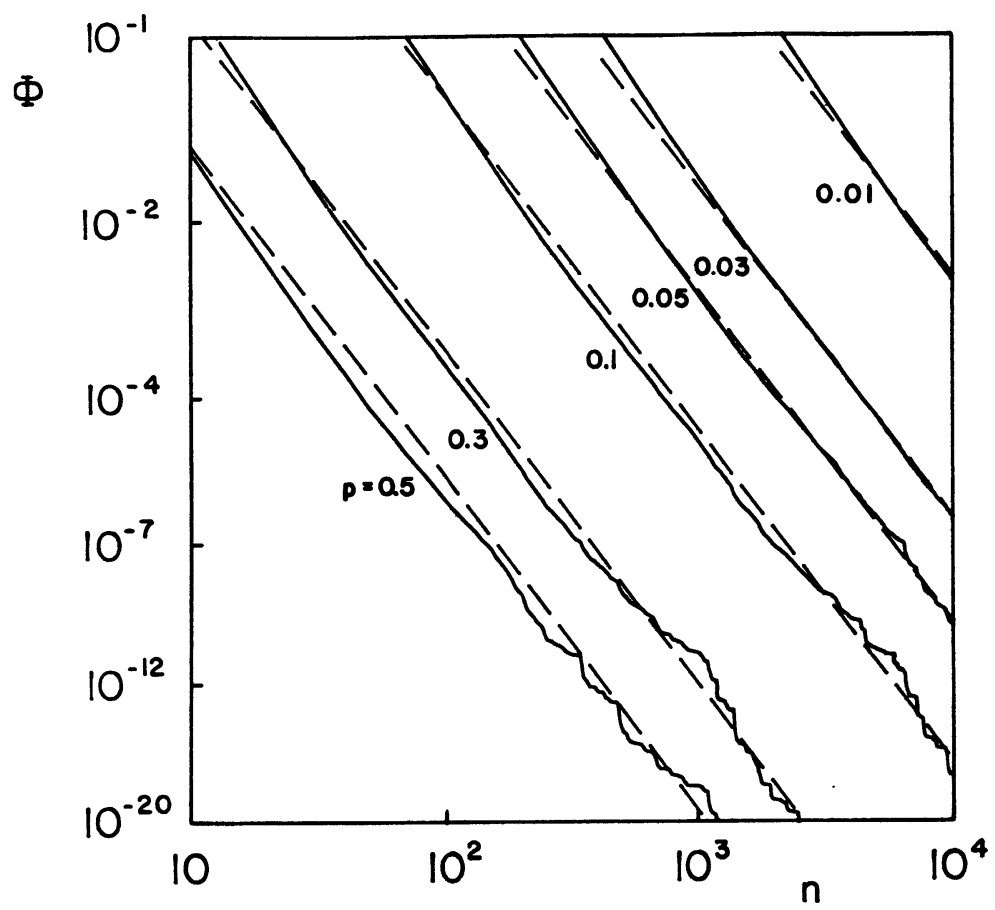

Fig. 3. - The decay law $\Phi$ for nearest-neighbour walks on the Sierpinski gasket of Euclidean dimension $d=2$. The trap concentration varies from $p=0.01$ to $p=0.5$. The scales of the drawing and the symbols are as in figure 2 .

\section{Conclusions.}

In this letter we have presented the numerically evaluated decays for trapping on structures of dimensions between $d=1$ and $d=2$, in an effort to find decays of the form of (1). An extension of previous approaches to fractals has given as exponent $\alpha$ in (1) the value $\tilde{d} /(\tilde{d}+2)$. The derivation was greatly facilitated by the use of the concept of compact exploration, as suggested by de Gennes. While in all cases the form of (1) is qualitatively obeyed, only in the one-dimensional case did the asymptotic value of $\alpha=1 / 3$ appear in a range amenable to experimental observation; in the other cases we could not get a value of $\alpha$ close to the predicted one, although we analysed the decay forms over twenty orders of magnitude. We thus doubt that for $\not{d} \neq 1$ the relation $\alpha=\tilde{d} /(\tilde{d}+2)$ could be observed experimentally.

\section{Acknowledgments.}

For providing them with preprints before publication, the authors are very thankful to Prof. Toulouse, Dr. Rammal and Dr. Vannimenus. Discussions with Prof. Alexander and Prof. Dressler and the support of the Fonds der Chemischen Industrie and of the Deutsche Forschungsgemeinschaft are gratefully acknowledged. The computations were made possible by a grant from the Rechenzentrum - ETH. 


\section{References}

[1] Movaghar, B., SAuer, G., Würtz, D. and Huber, D. L., Solid State Commun. 39 (1981) 1179, and J. Stat. Phys. 27 (1982) 473.

[2] Zumofen, G. and Blumen, A., Chem. Phys. Lett. 88 (1982) 63.

[3] Grassberger, P. and Procaccia, I., J. Chem. Phys. 77 (1982) 6281.

[4] Kayser, R. F. and Hubbard, J. B., Phys. Rev. Lett. 51 (1983) 79.

[5] Balagurov, B. Ya and Vaks, V. G., Zh. Exp. Teor. Fiz. 65 (1973) 1939 [English translation : Sov. Phys. JETP 38 (1974) 968].

[6] Seiferheld, U., Bässler, H. and Movaghar, B., Phys. Rev. Lett. 51 (1983) 813.

[7] Zumofen, G. and Blumen, A., Chem. Phys. Lett. 83 (1981) 372.

[8] Mandelbrot, B., The Fractal Geometry in Nature (W. H. Freeman, San Francisco) 1982.

[9] Alexander, S. and Orbach, R., J. Physique Lett. 43 (1982) L-625.

[10] Rammal, R. and Toulouse, G., J. Physique Lett. 44 (1983) L-13.

[11] Rammal, R., in : Common Trends in Particle and Condensed Matter Physics, Les Houches Proceedings (1983).

[12] Gefen, Y., Aharony, A. and AleXander, S., Phys. Rev. Lett. 50 (1983) 77.

[13] De Gennes, P.-G., C.R. Hebd. Séan. Acad. Sci. 296, Série II (1983) 881.

[14] Evesque, P., J. Physique, in press.

[15] Blumen, A., Klafter, J. and Zumofen, G., Phys. Rev. Rapid Commun., in press.

[16] Blumen, A. and Zumofen, G., J. Stat. Phys. 30 (1983) 487.

[17] Angles d'Auriac, J. C., Benoit, A. and Rammal, R., J. Phys. A, submitted.

[18] Weiss, G. H., Proc. Natl. Acad. Sci. 77 (1980) 4391.

[19] Blumen, A. and Zumofen, G., J. Chem. Phys. 77 (1982) 5127.

[20] De Gennes, P.-G., J. Chem. Phys. 76 (1982) 3316; 3322.

[21] LifshitZ, I. M., Adv. Phys. 13 (1964) 483.

[22] Tanaka, F., J. Phys. C 13 (1980) L1.

[23] Bender, C. M. and Orszag, S. A., Advanced Mathematical Methods for Scientists and Engineers (McGraw-Hill, New York) 1978.

[24] Zumofen, G., Blumen, A. and Klafter, J., to be published. 